\title{
Anorgasmia in anterior spinal cord syndrome
}

\author{
Aleksandar Berić, J Keith Light
}

\begin{abstract}
Three male and two female patients with anorgasmia and dissociated sensory loss due to an anterior spinal cord syndrome are described. Clinical, neurophysiological and quantitative sensory evaluation revealed preservation of the large fibre dorsal column functions from the lumbosacral segments with concomitant severe dysfunction or absence of the small fibre neospinothalamic mediated functions. These findings indicate a role for the spinothalamic system in orgasm.
\end{abstract}

$(\Im$ Neurol Neurosurg Psychiatry 1993;56:548-551)

Patients with paraplegia and tetraplegia may have transitory or permanent sexual dysfunction. ${ }^{1-3}$ This dysfunction is more common with more severe lesions. ${ }^{4}$ Reproductive functions may not be significantly altered in females. ${ }^{5}$ Males commonly have absent ejaculation and/or impaired spermatogenesis depending on the level of lesion. ${ }^{6} \mathrm{~A}$ lesion above T10 which does not interfere with either the sympathetic or the parasympathetic functions, usually results in less severe sexual dysfunction than lumbosacral lesions. Both sexes may develop anorgasmia, irrespective of the level of the spinal lesion. The surgical literature has documented that anteriolateral cordotomy, especially bilateral, results in sexual dysfunction, including anorgasmia. ${ }^{7-10}$ These reports are conflicting as the sexual dysfunction has not been systematically analysed and the pre-existing abnormalities for which the cordotomy was performed may have influenced the results. The bladder and bowel pathways travel along the anterolateral tracts. ${ }^{112}$ The precise role that the anterolateral system plays in sexual function, including orgasm, is unclear. There are no reports on the sacral sensory finding in patients with anorgasmia. The function of the large fibre system and dorsal columns has likewise not been precisely assessed in these patients.

We interviewed all patients seen during 1990-91 at the Division of Restorative Neurology and Human Neurobiology, Baylor College of Medicine, who had dissociated sensory loss in the lumbosacral segments as established with quantitative sensory assessment.

Material and methods

Ninety six patients were assessed clinically and by quantitative sensory testing during a
16 month period. Three male and two female patients had dissociated sensory loss in the lumbosacral segments without a peripheral nerve or lumbosacral cord lesion. Their ages ranged from 20 to 56 years.

The patients' sexual functions were assessed clinically with special emphasis on the presence or absence of orgasm. Clinical sensory examination was performed followed by quantitative sensory evaluation of vibratory perception, temperature and thermal pain in the lumbosacral segments. All the patients were tested in a sound-proof, temperature controlled $\left(22^{\circ} \mathrm{C}\right)$ room with the patient supine to provide optimal relaxation and concentration during the test procedures. Vibratory threshold measurements were obtained with a vibrameter (Somedic AB Stockholm) designed according to Goldberg and Lindblom. ${ }^{13}$ The probe was placed over the first metatarsal bone or over the anterior surface of the tibia. The vibratory threshold was determined according to the methods of limits. Three successive measurements of threshold were averaged for each test site. Temperature perception and cold and heat pain perception were tested with a Marstock thermal stimulator (Somedic AB) based on the technique by Fruhstorfer et al. ${ }^{14}$ The thermode stimulating surface, consisting of 36 Peltier elements, was either warmed or cooled depending on the direction of the applied current. A thermocouple attached to the thermode measured the change in skin temperature. The thermode was placed laterally over the dorsum of the feet, and thresholds for warm, cool, cold pain and heat pain recorded. In addition to the standard stimulation sites, temperature and thermal pain perception were assessed over the S3 and S4 dermatomes in the gluteal fold.

Neurophysiological assessment included recording lumbosacral and cortical somatosensory evoked potentials according to a technique described previously. ${ }^{15}$ Cortical SEPs were recorded using silver-silver chloride electrodes placed over the modified $\mathrm{Cz}$ point $(1.5 \mathrm{~cm}$ behind $\mathrm{Cz}$ ), C3' and C4' points ( 1 to $1.5 \mathrm{~cm}$ behind $\mathrm{C} 3$ and $\mathrm{C} 4$ ) referenced to $\mathrm{Fz}$ (International 10/20 system). Electrode impedance was kept below $5 \mathrm{KOhm}$. A 0.5 ms rectangular pulse was delivered to the tibial nerve at the popliteal fossa by a $2 \mathrm{~cm}$ diameter disc cathode and $5 \mathrm{~cm}$ square lead plate anode positioned over the patella. Two intensities of stimulus strength were used, one to produce a maximal $\mathrm{H}$ response, and the other to produce a maximal $M$ response of the soleus muscle. Pseudo-random stimulation was used with stimuli delivered during 
the quiescent phase of the ECG by delaying the trigger 300-500 ms after the QRS complex. At least two repeatable averages of 128 responses were obtained with an analysis time of $200 \mathrm{~ms}$. The Viking I system (Nicolet Biomedical) was used for amplification, averaging and analysis. Analog filters were $5 \mathrm{~Hz}$ to $1 \mathrm{kHz}$. Lumbosacral somatosensory evoked potentials (LSEPs) were recorded simultaneously by Beckman surface electrodes placed at $\mathrm{T} 12, \mathrm{~L} 2, \mathrm{~L} 4$, and $\mathrm{S} 1$ spinous processes referenced to an electrode at T6. Pairs of Beckman electrodes were placed over the soleus muscles to monitor $H$ and $M$ responses. Analog filters for $H$ and $M$ responses, as well as for LSEPs were from $10 \mathrm{~Hz}$ to $3 \mathrm{kHz}$.

In addition, pudendal cortical SEP was recorded in one patient. Pudendal SEP was recorded with surface cup electrodes placed at $\mathrm{Cz}^{\prime}(1.5 \mathrm{~cm}$ posterior to $\mathrm{Cz}$ according to the 10-20 International EEG system) referenced to $\mathrm{Fz}$ electrode. The stimulus was a $0 \cdot 2$ ms rectangular pulse delivered to the clitorial nerve by the bipolar bar electrode (Teca Inc). The stimulus intensity was adjusted to be sufficient to elicit a clinical bulbocavernosus reflex. The amplification and averaging was accomplished with DISA 1500 System. Bandwidth was $10 \mathrm{~Hz}$ to $1 \mathrm{kHz}$ with an analysis time of $100 \mathrm{~ms}$. Two reproducible averages of 128 stimuli were obtained and analysed for latency and amplitude.

Video cystometry was performed on all five patients. A number 10 French double lumen Porges catheter was introduced transurethrally into the bladder. One channel was connected to a Statham transducer and the other to an infusion pump. The bladder was filled at the rate of $60 \mathrm{cc}$ per minute to a volume of $700 \mathrm{cc}$ with the patient in the left oblique or supine position. The urodynamic results were recorded on a Dantec 5500 urograph. The results were interpreted according to International Continence Society guidelines.

\section{Results}

All five patients complained of anorgasmia regardless of the aetiology of their dissociated sensory loss.

Patient 1, a 29 year old female suffered multiple stab wounds to the chest and back in March 1989 and developed hypotensive episodes during surgery with clamping of the aorta. Post operatively she became deeply paraparetic with absence of temperature perception in the lower trunk and lower extremities. Clinical examination revealed mild hypertonia in the lower extremities with symmetrical deep tendon reflexes. Clonus was absent but the Babinski sign was present bilaterally. Pudendal SEP revealed a normal W waveform with a normal P1 latency of 31 ms. The sensory threshold to electrical stimulation of the clitorial nerve was normal. The patient has been unable to obtain an orgasm since injury despite normal libido, sexual activity with partner and sexual counselling.
Patient 2, a 24 year old white male, sustained a C7 spinal cord injury following a car accident in August 1984. He was left tetraplegic. During the following months, however, he regained some sensation and motor function in the lower extremities but still complained of spasticity and required braces and crutches to walk. Clinical examination revealed increased deep tendon reflexes in both the upper and lower extremities with bilateral Hoffman sign, unsustained clonus at the knee and ankle level and a Babinski sign bilaterally. Although the patient has a normal libido and erection, he denies ejaculations and orgasms since his injury.

Patient 3, a 57 year old male, was injured in December 1985 in a tractor accident causing a complete C6 tetraplegia. Clinical examination showed exaggerated deep tendon reflexes bilaterally in the lower extremities with increased tone and occasional flexor and extensor spasms. A sustained right ankle clonus was present with bilateral Babinski sign. The patient is able to obtain a reflex erection which is short-lasting and unsatisfactory for sexual intercourse. He has not had ejaculation or orgasms since injury.

Patient 4, a 20 year old male, sustained a C5-6 SCI in October 1989 during a football game resulting in initial complete paralysis below the lesion. Sensory and motor functions later improved in the proximal arm and shoulder. The patient also regained some, although different, sensation related to urgency to urinate. $\mathrm{He}$ also complains of a burning pain in the buttocks and has occasional spasms in the lower extremities, usually triggered by volitional efforts to move his legs. Clinical examination revealed absent voluntary control in the lower extremities with symmetrical deep tendon reflexes and a bilateral Babinski sign. The patient denies ejaculation and orgasm, although he is able to obtain erections sufficient for sexual intercourse.

Patient 5, a 44 year old female with a chief complaint of constant burning pain in the thoracolumbar back, buttocks, feet and ankles, was diagnosed in 1980 with arteriovenous malformation (AVM) involving the $\mathrm{T} 7$ to $\mathrm{T} 9$ radicular arteries. She had two embolisation procedures in 1980. Between 1981-83 she noticed increasing weakness of her legs. Clinical examination showed that the deep tendon reflexes were symmetrical in the upper and lower extremities with the Babinski sign present bilaterally. The patient has a normal libido and a history of anorgasmia since 1981 .

All 5 patients showed absent pin prick and temperature sensation below the spinal lesion. Quantitative sensory evaluation confirmed absent temperature perception in four patients. Patient 3, however, retained some perception of cold, albeit altered. Thermal pain was also absent in patients 2,3 and 4, while in patients 1 and 5 some heat pain perception was retained, but with a significantly higher level than normal. LSEPs were normal in all patients suggesting that the peripheral 
sensory neuron and lumbosacral cord were intact. Patients were at least 18 months after the onset of dysfunction and they were not taking any antidepressant or neuroleptic medication. They were all sexually active before injury and able to obtain orgasm. Patients 1 and 2 reported anorgasmia as one of their major complaints. All the patients went through several levels of rehabilitation programmes, including sexual counselling, and were considered fully rehabilitated in accordance with the level of their disability. Only Patient 4 was considered severely disabled.

\section{Discussion}

Sexual dysfunction, including anorgasmia may be a complication of treatment with medication such as neuroleptics and antidepressants. ${ }^{16-18}$ Decrease in dose or discontinuation of the offending medication usually resolves the anorgasmia. Trauma to the nervous system may also result in transitory sexual dysfunction. This, however, may be permanent in patients with spinal cord injury, especially those with complete lesions. As our patients were able to obtain orgasm before injury and were not taking any medication that could induce anorgasmia, it can be concluded that their sexual dysfunction is the direct result of the trauma. Sexual dysfunction following spinal cord injury has been studied in detail by several investigators, ${ }^{1-6}$ 19-22 where it has been shown that the ability to obtain an erection is related to the intactness of the sacral segments, ${ }^{22}{ }^{23}$ whereas the male reproductive function, especially spermatogenesis, is related to the lower lumbar segments. ${ }^{6}$ It has been suggested, especially in females, that in the presence of anorgasmia other erogenic zones can be developed as a replacement for the major genital zone. ${ }^{2}$ However, anorgasmia continues to be a significant problem in both sexes, especially with high tetraplegia. There are, however, other causes of anorgasmia in patients with spinal cord injury unrelated to the neurogenic injury. These include depression, difficulty with positioning and fear of urinary incontinence during intercourse as a result of the associated neurogenic bladder. Libido may also be decreased for the same reasons. These important factors are unlikely to play a role in our patients as they all had normal libido and had graduated from a sexual rehabilitation programme. In addition, their partners had been educated about urinary incontinence and the necessity of performing clean catheterisation before intercourse. This is in contrast to stroke patients where a decrease in libido and sexual function may be the result of fear of inducing hypertension and thus the possibility of restroke. ${ }^{24}$ It is therefore reasonable to conclude that anorgasmia in our patients was the result of neurological dysfunction only. The topic of sexual dysfunction in neurological disease has been addressed from many aspects including psychosocial, psychiatric, disability and reha- bilitation, but the question of underlying neurogenic dysfunction has been neglected. As pointed out by Brindley, ${ }^{20}$ anorgasmia has not been discussed in relation to the anatomy of the spinal cord lesion, for example, anterior, posterior, or lateral, which is a major part of our study.

The question of anorgasmia is a difficult and sensitive issue. Orgasm is a subjective sensation, and we have relied on patients' reports regarding its presence or absence. Due to the fact that all patients were and are sexually active and have had orgasm in the past, we accepted their reports of anorgasmia as reliable. In addition, none of the patients were in litigation or were considered unreliable during their extensive assessments.

A precise assessment would help in the understanding, classification and prognosis of the anorgasmia. This study suggests that anorgasmia in patients with dissociated sensory loss and severe or complete dysfunction of the spinothalamic system, is long lasting and possibly permanent. Only patients with an intact sacral spinal cord were studied to evaluate possible spinal cord pathways involved with orgasm. It is possible that some descending facilitation is necessary as only $5-10 \%$ of patients with spinal cord injury are able to obtain ejaculation, regardless of the degree of incompleteness. Other stimuli such as vibration, electrical stimulation or intrathecal physostigimin may also trigger ejaculation in patients with spiral cord injury who otherwise would be unable to ejaculate. All of our patients had dissociated sensory loss with preserved dorsal column modalities of sacral sensation, making it unlikely that ejaculation would be unnoticed. A dissociation between ejaculation and orgasm is possible ${ }^{2526}$ but rare in the non-spinal cord injury population. Interestingly, non-ejaculatory orgasm in males is also possible, ${ }^{25}$ further complicating the issue. It is necessary to have preserved ascending functions allowing impulses from the lumbosacral cord to reach the brain for orgasm to occur, regardless of the mechanism of orgasm. As we did not assess whether strong peripheral stimulation would elicit ejaculation in our patients or if they were able to achieve orgasm at that time, we postulate that descending facilitation through the anterolateral quadrant was probably involved in addition to the presence of ascending spinothalamic dysfunction. We cannot specify if any anterolateral spinal tract, other than the neospinothalamic system, is important for orgasm. Based on good motor control and muscle strength in patient 1 and the unilateral good motor control in patient 2 , the descending motor system, or pathways travelling along them are unlikely to be relevant. Bladder function itself does not appear to add to the understanding of the sexual dysfunction because it is multifactorial (influence of sphincterotomy, recurrent infections, etc.) and in our patients was either normal or abnormal, being either hyperreflexic or even areflexic in one patient. As the patients did not have any local lumbosacral dysfunction, 
their reflex functions were preserved, including presumably the ejaculation reflex in males as well as lubrication in females.

The five patients described with anorgasmia all had severe or complete absence of small fibre spinothalamic functions but preserved larger fibre mediated-dorsal column functions. This finding implies that, at least in a subset of patients, all the tests assessing large fibre functions, such as bulbocavernosus reflex, electromyography or somatosensory evoked potentials usually used for impotence assessment, are not specific for anorgasmia. A severe spinal lesion frequently involves both the anterior and posterior aspects of the spinal cord. Due to the extent of the lesion, abnormal SEPs may exhibit non-specific correlation with the presence of anorgasmia. With peripheral nerve lesions, as in polyneuropathies, small and large fibre dysfunction are frequently both present and testing of functions mediated through the large fibres might correlate with a dysfunction which is presumably mediated by the small fibres. ${ }^{27} 28$ In erectile dysfunction the more specific tests for autonomic and small fibre functions, such as sympathetic skin response of the perineum produce abnormal results more often than tests of large fibre function, for example, bulbocavernosus reflex. ${ }^{29}$ However, large fibre dysfunction may contribute to erectile dysfunction, secondary to the lack of continuous peripheral input necessary for maintaining an erection. This is why we excluded patients with peripheral neuropathy and low thoracic or lumbosacral lesions, thus enabling us to assess the contribution of the anterolateral tract function to the anorgasmia. It is reasonable to conclude that preservation of the spinothalamic function is necessary for orgasm to occur. There are several unanswered questions. What kind of sensation would male patients with anorgasmia due to anterior spinal cord syndrome have if ejaculation could be obtained by strong peripheral stimulation? There is controversy as to whether patients with spinal cord injury experience true orgasm or only feel some different but pleasurable sensation. Our patients are able to feel sensations from the perineum due to preserved dorsal columns but are still unable to achieve orgasm. As strong artificial stimulation was tried by some of the patients without success, preservation of the dorsal columns does not appear to be enough to preserve orgasm following bilateral lesions of the anterolateral tracts.

We are indebted to the personnel of the Division of Restorative Neurology and Human Neurobiology, Baylor College of Medicine, Houston, Texas, for excellent technical assistance.
Supported in part by The Vivian L Smith Foundation for Restorative Neurology, Houston, Texas.

1 Bors E, Comarr E. Neurological disturbances of sexual function with special reference to 529 patients with spinal cord injury. Urol Surv 1960;10:191-222.

2 Gutmann L. The married life of paraplegics and Guttmann L. The married life of

3 Munro D, Horne HW Jr, Paull DPP. The effect of injury to the spinal cord and cauda equina on the sexual potency of men. $N E \mathcal{F} M \quad 1948 ; 239: 903-11$.

4 Siösteen A, Lundqvist C, Blomstrand C, Sullivan L, Sullivan M. Sexual ability, activity, attitudes and satisfaction as part of adjustment in spinal cord-injured subjects. Paraplegia 1990;28:285-95.

5 Bérard EJJ. The sexuality of spinal cord injured women: physiology and pathophysiology. A review. Paraplegia 1989;27:99-112.

6 Chapell PA, Roby-Brami A, Yakovleff A, Bussel B. Neurological correlations of ejaculation and testicular size in men with a complete spinal cord section. $\mathcal{F}$ Neurol Neurosurg Psychiatry 1988;51:197-202.

7 White JC. Cordotomy: Assessment of its effectiveness and suggestions for its improvement. Clin Neurosurg 1966;13:1-9.

8 White JC, Sweet WH, Hawkins R, Nilges RG. Anterolateral cordotomy: results, complications and causes of failure. Brain 1950;73:346-66.

9 Rosomoff $\mathrm{HL}$. Bilateral percutaneous cervical radiofrequency cordotomy. $\mathcal{F}$ Neurosurg 1969;31:41-46.

10 Lipton S. Percutaneous cordotomy. In: Wall PD, Melzack R, eds. Textbook of pain. Edinburgh: Churchill Livingstone $1989 ; 832-9$.

11 Nathan PW, Smith MC. The centripetal pathway from the bladder and urethra within the spinal cord. $f$ Neurol Neurosurg Psychiatry 1951;14:262-80.

12 Nathan PW, Smith MC. Spinal pathways subserving defecation and sensation from the lower bowel. $\mathcal{f}$ Neurol Neurosurg Psychiatry 1953;16:245-56.

13 Goldberg JM, Lindblom U. Standardized method of determining vibratory perception thresholds for diagnosis and screening in neurological investigation. $f$ Neurol Neurosurg Psychiatry 1979;42:793-803.

14 Fruhstorfer H, Lindblom U, Schmidt WG. Method for quantitative estimation of thermal thresholds in patients. F Neurol Neurosurg Psychiatry 1976;39:1071-5.

15 Berić A, Dimitrijevic MR, Lindblom U. Cortical evoked potentials and somatosensory perception in chronic spinal cord injury patients. I Neurol Sci 1987;80: 333-42.

16 Monteiro WO, Noshirvani HF, Marks IM, Lelliott PT. Anorgasmia from clomipramine in obsessive-compulsive disorder. A controlled trial. Br $\mathcal{F}$ Psychiat 1987;151: 107-12.

17 Nurnberg AU, Levine PE. Spontaneous remission of MAOI-induced anorgasmia. Am $\mathcal{f}$ Psychiat 1987;144: 805-7.

18 Shen WW, Sata LS. Inhibited female orgasm resulting from psychotropic drugs. F Reprod Med 1990;35:11-14.

19 Beretta G, Chelo E, Zanollo A. Reproductive Aspects in Spinal Cord Injured Males. Paraplegia 1989;137: 113-8.

20 Brindley GS. Physiology of erection and management of paraplegic infertility. In: Hargreave TB, ed. Male infertility. Berlin: Springer-Verlag, 1983:509-61.

21 Kennedy S, Over R. Psychophysiological assessment of male sexual arousal following spinal cord injury. Arch Sex Behav 1990;19:15-27.

22 Yalla SV. Sexual dysfunction in the paraplegic and quadriplegic. In: Bennett AH, ed. Management of male plegic. In: Bennett AH, ed. Management of male Impotence, international perspectives in urology,

23 Brindley GS, Sauerwein D, Hendry WF. Hypogastric plexus stimulators for obtaining semen from paraplegic man. Brit $\mathcal{F}$ Urol 1989;64:71-77.

24 Mouga TN, Lawson TS, Inglis J. Sexual dysfunction in stroke patients. Arch Phys Med Rehabil 1986;67:19-22.

25 Dunn ME, Trost JE. Male multiple orgasms: a descriptive study. Arch Sex Behav 1989;18:377-87.

26 Rosenbaum JF, Pollack MH. Anhedonic ejaculation with desipramine. Int $\mathcal{f}$ Psychiatry Med 1988;18:85-8.

27 Tackman W, Porst $H$, van Ahlen $H$. Bulbocavernosus reflex latencies and somatosensory evoked potentials after pudendal nerve stimulation in the diagnosis of impotence. $₹$ Neurol 1988;235:219-25.

28 Gerstenberg TC, Nordling J, Hald T, Wagner G. Standardized evaluation of erectile dysfunction in 95 consecutive patients. $¥$ Urol 1989;141:857-62.

29 Ertekin C, Ertekin N, Almis S. Autonomic sympathetic nerve involvement in diabetic impotence. Neurourol Urodynamics 1989;8:589-98. 